\title{
SGEF is overexpressed in prostate cancer and contributes to prostate cancer progression
}

\author{
HONGTAO WANG, RUIQIN WU, LAN YU, FEIMA WU, SHANHU LI, YALI ZHAO, \\ HAILIANG LI, GUOLAN LUO, JIAN WANG and JIANGUANG ZHOU
}

Laboratory of Medical Molecular Biology, Beijing Institute of Biotechnology, Beijing, P.R. China

Received April 9, 2012; Accepted May 30, 2012

DOI: 10.3892/or.2012.1917

\begin{abstract}
The purpose of this study was to investigate the potential roles of the $\mathrm{SH} 3$-containing guanine nucleotide exchange factor (SGEF) in human prostate cancer. Experimental data showed that SGEF was overexpressed in human prostate cancer cells and specimens. The reduction of SGEF expression through an SGEF-targeting siRNA in androgen-independent C4-2 and C4-2B cells suppressed both anchorage-dependent and anchorage-independent growth. In addition, the androgen receptor (AR) antagonist bicalutamide further strengthened this inhibitory effect due to the suppression of the elevated AR transactivation after knockdown of SGEF. Collectively, our results provide the first demonstration that SGEF is a novel promoter of human prostate cancer progression and development.
\end{abstract}

\section{Introduction}

SGEF was initially identified in a screen for androgen-responsive genes in human prostate cancer cells (1). SGEF contains $\mathrm{N}$-terminal proline-rich domain, $\mathrm{DH}$ domain in tandem with a $\mathrm{PH}$ domain and C-terminal SH3 binding domain. The DH-PH module is essential for GEF activity of most DBL family members. Proline-rich domain and SH3 binding domain are assumed to be associated with protein-protein interactions and modulation of exchange activity of SGEF. SGEF supports the exchange of GDP for GTP on Rho G and is the first identified mammalian RhoGEF that promotes macropinocytosis (2). SGEF has been reported to be involved in the uptake of

Correspondence to: Dr Jianguang Zhou and Dr Jian Wang, Beijing Institute of Biotechnology, 27 Taiping Road, Haidian, Beijing 100850, P.R. China

E-mail: zhou.jianguang@yahoo.com.cn

E-mail: newgeno2003@yahoo.com.cn

Abbreviations: SGEF, SH3-containing guanine nucleotide exchange factor; PCa, prostate cancer; AI, androgen independence; AR, androgen receptor; $\mathrm{BPH}$, benign prostatic hyperplasia; TMA, tissue microarray; GTPase, guanosinetriphosphatase; GEF, guanine nucleotide exchange factor

Key words: SGEF expression, prostate cancer, androgen receptor, AR transactivation, bicalutamide
Salmonella by epithelial cells by stimulating the formation of the surfaces phagocytic cups (3). It is also reported that SGEF can promote leukocyte trans-endothelial migration by regulating endothelial apical cup assembly (4). All the data reported have suggested that SGEF play a role on various physiological and pathological situations in association with phagocytosis or the uptake of particulate material. In contrast, the functions of SGEF in human cancer still remain unknown.

Prostate cancer $(\mathrm{PCa})$ remains the most commonly diagnosed and the second leading cause of cancer-related death in men in the western world (5). Although early stage PCa is responsive to androgen withdrawal, it ultimately progresses to AI and there is no curative therapy available at present. Improved understanding of the molecular events of prostate cancer progression will contribute to developing new therapeutic approaches.

In this study, we explored the expression and potential roles of SGEF in human prostate cancer. Our studies revealed that SGEF was overexpressed in human specimens and AI prostate cancer cells. Moreover, the growth of AI prostate cancer cell lines C4-2 and C4-2B was suppressed by knockdown of SGEF and this inhibitory effect can be enhanced by bicalutamide, which is known as AR antagonist. Collectively, these data identify SGEF as a novel potential promoter of human prostate cancer progression.

\section{Materials and methods}

Cell culture. C4-2 and C4-2B cell lines were generous gifts from L.W. Chung (Emory University, USA), cultured in RPMI-1640 (Gibco, Paisley, UK) containing 10\% fetal bovine serum (FBS; Hyclone, Logan, UT, USA) at $37^{\circ} \mathrm{C}$ in $5 \% \mathrm{CO}_{2}$. Where indicated, cells were treated with $1 \mathrm{nM}$ R1881 (methyltrienolone) in fresh phenol red-free RPMI-1640 with 5\% dextran/charcoal absorbed fetal bovine serum (cFBS; Hyclone) or $10 \mu \mathrm{M}$ bicalutamide in culture medium with $10 \%$ FBS. For shRNA experiments, C4-2 or C4-2B cell lines were infected with lentivirus derived from pU6-vshRNA-CMV-GFP (GeneChem Co., Shanghai, China), containing the shRNA human SGEF sequence 5'-GGAA TCTTGTGACAATGAAGA-3'.

Western blot analysis. Western blot analysis was performed as described previously (6). Rabbit anti-SGEF (Sigma, St. Louis, MO, USA), mouse actin antibody (Santa Cruz Biotechnology, Inc., Heidelberg, Germany), rabbit anti-AKT and rabbit anti- 
AKT/phosphor Ser473 (Cell Signaling Technology, Inc., Danvers, MA, USA) were used as primary antibodies. Proteins were visualized by using the enhanced chemiluminescence kit (Pierce) after incubation with anti-mouse or anti-rabbit HRP conjugated secondary antibodies (Zhongshan Golden Bridge Biotechnology Co., Ltd.).

RT-PCR. Total RNA was extracted from cells using TRIzol reagent (Invitrogen Life Technologies, CA, USA). Reverse transcription was performed with $1 \mu \mathrm{g}$ RNA using the First Strand Synthesis System kit (Toyobo, Japan) according to the manufacturer's instruction. The following primers were used for PCR amplification: 5'-ACTCGGTGTTGCTCCTCCC-3' and 5'-GGCTCCTATGTACCGTCCTG-3' for SGEF; 5'-GAG CTACGAGCGCCTGACG-3' and 5'-CCTAGAAGCTTTG CGGTGG-3' for $\beta$-actin.

Growth rate assay. To obtain growth curves, 1,500 cells were seeded in 96-well plates. Cell growth was examined at indicated time-points using 3-(4,5-dimethylthiazol-2-yl)-2,5-diphenyltetrazolium bromide (MTT) assays according to the manufacturer's directions (Amresco). Each experiment was repeated independently four times.

Colony formation assay. For the colony-formation assay, 2,000-6,000 cells were seeded in 6-well plates. After 12 days, cells were washed in PBS and colonies were fixed by methanol and stained with $0.1 \%$ crystal violet (Sigma). The colonies containing $>50$ cells were counted.

Soft agar assay. A total of 2,000-6,000 cells were suspended in $2 \mathrm{ml}$ of $0.17 \%$ low melting agarose (Difco Laboratories) dissolved in RPMI-1640/10\% FBS and plated on the top of $3 \mathrm{ml}$ underlayer of $0.5 \%$ agarose in the same medium in 6 -well culture plates. After 3 weeks of incubation at $37^{\circ} \mathrm{C}$ in $5 \% \mathrm{CO}_{2}$, the colonies containing $>15$ cells were counted.

Luciferase reporter assays. C4-2 and C4-2B cells were seeded in 24-well plates $\left(5 \times 10^{4}\right.$ cells per well) and transfected with 300 ng PSA-luc reporter gene plasmids, $100 \mathrm{ng}$ pCMV-2B or pCMV-2B-SGEF (PGPU6/GFP/Neo-si-NC or PGPU6/GFP/ Neo-si-SGEF) plasmids and $30 \mathrm{ng}$ of pCMV- $\beta$-gal plasmids (internal control) using the Lipofectamine 2000 reagent (Invitrogen). After transfection, cells were treated with $1 \mathrm{nM}$ $\mathrm{R} 1881$ or vehicle for $24 \mathrm{~h}$. Then luciferase activity was measured using luciferase assay system (Promega Corp., Madison, WI, USA) normalized by $\beta$-galactosidase activity. All transfection experiments were carried out in triplicate wells and repeated three times.

Immunohistochemistry. Immunohistochemistry was performed as described previously using TMA (Chaoying Biotechnology Co., Xi'an, China) including 75 prostate specimens (10 benign prostate tissues, $19 \mathrm{BPH}$ tissues and 46 tumor tissues) (6). SGEF expression was scored blindly by staining intensity in each biopsy core as being weak, moderate or strong.

Statistical analysis. Statistical evaluation was performed using the statistical software SAS/STAT. $\mathrm{p}<0.01$ was considered statistically significant.

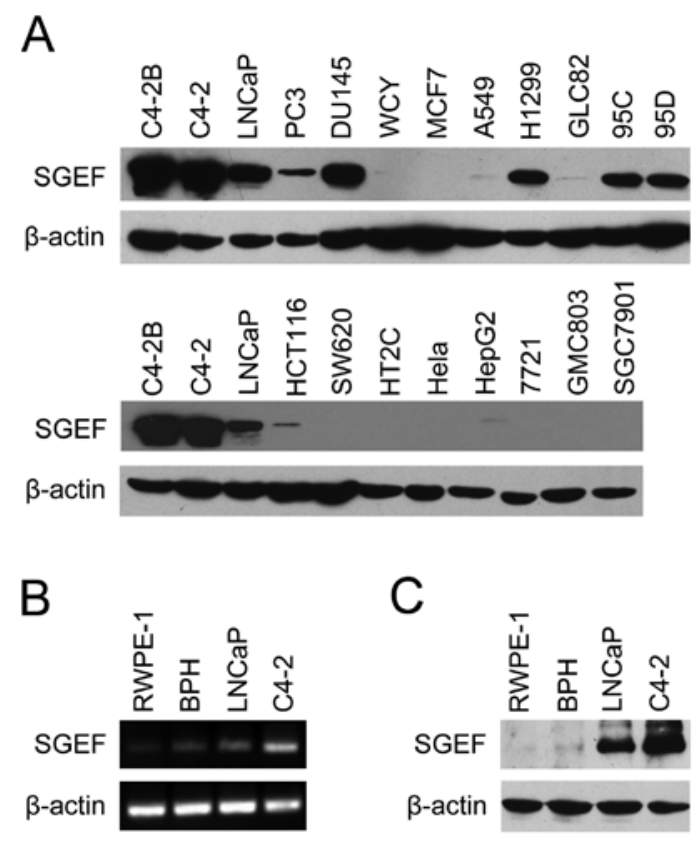

Figure 1. Expression pattern of SGEF analyzed in various cancer cell lines and in the cell lines representing the different status of prostate cancer progression. (A) SGEF expressions were found to be limited to the prostate cancer cell lines (C4-2B, C4-2, LNCaP, PC3 and DU145 cell lines) and part of the lung cancer cell lines (A549, H1299, GLC82, 95C and 95D cell lines) and hardly detected in other cancer cell lines. (B and C) SGEF expressions were found to be positively associated with prostate malignant progression in both $\mathrm{mRNA}$ and protein levels.

\section{Results}

Expression analysis of SGEF in human cancer cells. We first examined the expression pattern of SGEF protein in various cancer cell lines by western blot analyses. As shown in Fig. 1A, the expression of SGEF was restricted to the prostate cancer cell lines $(\mathrm{C} 4-2 \mathrm{~B}, \mathrm{C} 4-2, \mathrm{LNCaP}, \mathrm{PC} 3$ and DU145) and part of the lung cancer cell lines (A549, H1299, GLC82, 95C and 95D), and absent or very low levels in breast cancer (MCF10 and WCY), colon cancer (HCT116, SW620 and HT29), cervical cancer (HeLa), liver cancer (HEPG2 and 7721) and gastric cancer (GMC803 and SGC7901). Moreover, all prostate cancer cell lines, except for PC3, had a higher level of SGEF than that of lung cancer. These results indicated that SGEF may be closely related to prostate cancer. Then RT-PCR and western blot analyses were performed to further analyze the expression patterns of SGEF in prostate cancer cells. As shown in Fig. 1C, SGEF protein was found to be absent or at a very low level in normal prostate epithelial cell line RWPE-1 and BPH cell line, moderate level in androgen-dependent prostate cancer cell line LNCaP, and of the highest level in androgen-independent cell line C4-2. The results of RT-PCR (Fig. 1B) were consistent with that of western blotting. These results further showed that SGEF expressions were positively associated with prostate cancer malignant progression.

SGEF expression is elevated in human prostate cancer specimens. To validate the clinical relevance of SGEF expression with human prostate cancer progression, we determined the expression of SGEF in human PCa specimens utilizing TMA 


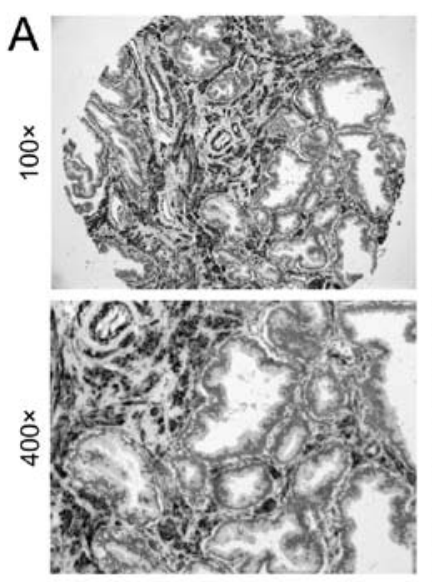

Normal

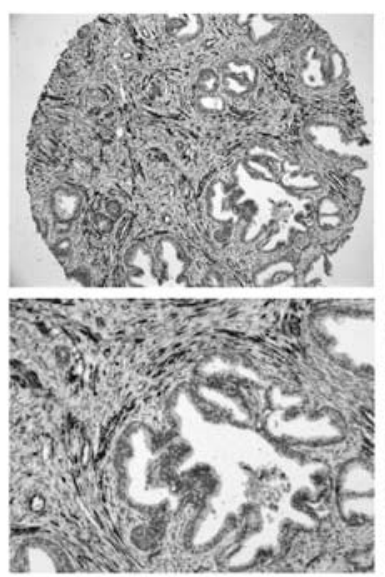

$\mathrm{BPH}$

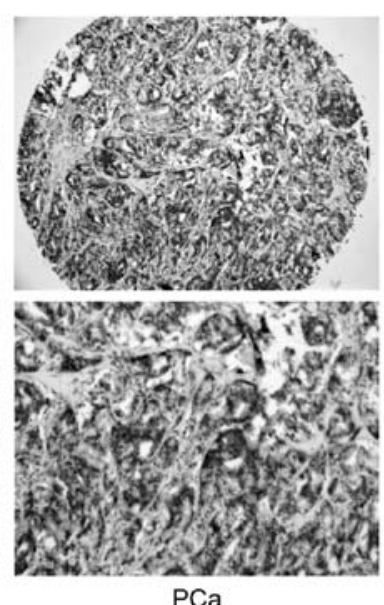

B

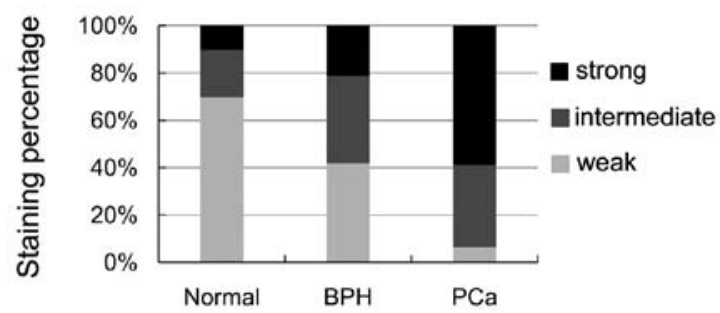

Figure 2. The expression of SGEF is increased in human prostate cancers. (A) Representative images of each normal prostate, BPH and PCa of the stained human prostate TMA. SGEF expression was detected by immunohistochemical analysis with anti-SGEF antibody at 1:40 dilution. (B) Statistical analysis of SGEF expression in each group of normal, BPH, PCa samples of the stained human prostate TMA in terms of staining intensity. Staining intensity was evaluated independently by two pathologists with weak, intermediate and strong staining for each sample.

containing 10 normal tissues, $19 \mathrm{BPH}$ tissues and 46 tumor tissues through immunohistochemistry. Fig. 2A shows the representative images of SGEF expression from human prostate TMA samples. As shown in Fig. 2B, the strong staining frequency of SGEF significantly increased in the epithelial cells of tumor samples $(58.7 \%)$ in comparison to normal prostate samples $(10 \%)$ and BPH samples (21.1\%). Compared with normal prostate samples (70\%) and BPH samples (42.1\%), the weak staining frequency of SGEF notably decreased in the epithelial cells of tumor samples (6.5\%). The TMA data suggested that SGEF expression was elevated and associated with the progression of prostate cancer.

Reducing the expression of SGEF inhibits the growth of prostate cancer cells. In order to investigate the potential role of SGEF in human prostate cancer cells growth, $\mathrm{C} 4-2$ and $\mathrm{C} 4-2 \mathrm{~B}$ cells were infected with lentivirus expressing an SGEF-targeted siRNA. Western blot assays showed that SGEF protein was sharply reduced (Fig. 3A). MTT assays were used to examine the correlation between knockdown of SGEF and the growth ability of C4-2 and C4-2B. The cell growth curves showed that silencing the expression of SGEF suppressed the growth of C4-2 and C4-2B cells (Fig. 3B). Then, colony formation assays and soft agar assays were respectively performed to determine the effect of SGEF reduction on the viability and anchorage-independent growth ability of prostate cancer cell. As data showed, C4-2 and C4-2B cells of the reduced SGEF displayed significantly decreased clonogenecity and anchorageindependent growth ability respectively, regardless of the cell number seeded (Fig. 3C and D). These results suggested that endogenous SGEF had positive roles to human prostate cancer cells growth.

SGEF suppress AR transactivation. It was reported that Vav3 protein, another Rho GTPase GEF similar to SGEF, contributed to prostate cancer cell growth through enhancing AR transactivation. Hence, we assumed that SGEF might function in the same way. To verify this supposition, FLAG-tagged SGEF or SGEF siRNA plasmid was transiently transfected into C4-2 and C4-2B cells along with PSA-Luc reporter construct in the presence of R1881 or not to examine the potential effects of SGEF on the AR transactivation. As shown in Fig. 4A, overexpression of SGEF decreases AR transactivation in an androgen-dependent manner. Consistent with the results of the SGEF overexpression, knockdown of SGEF increases AR transactivation in two cell lines (Fig. 4B). Taken together, these results indicated that SGEF could suppress AR transcriptional activity, but not increase its transcriptional activity.

$A R$ antagonist bicalutamide potentiates inhibitory effects of reduced SGEF on the growth of prostate cancer cells. The previous data indicated that knockdown of SGEF inhibited the growth of C4-2 and C4-2B, but augmented the transcriptional activity of AR. Hence, it is believed that the inhibitory effect of decreasing the SGEF expression on the growth of C4-2 and C4-2B cells could be further strengthened by the AR antagonist, which could repress the AR transactivation. As expected, bicalutamide, an AR antagonist, further enhanced the suppression of the clonogenicity and anchorage-independent growth of C4-2 and C4-2B cells by silencing the SGEF expression. For 
A

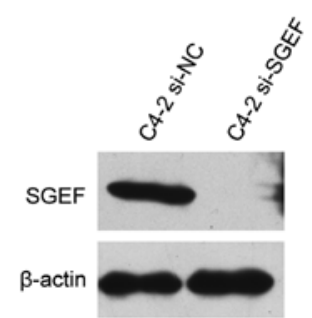

B

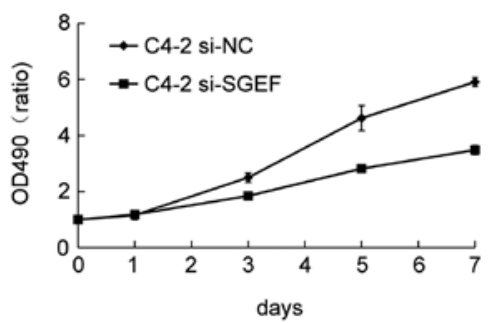

C

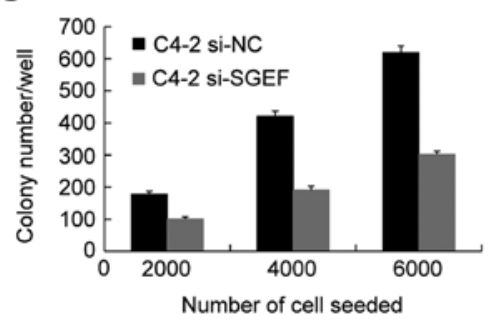

D

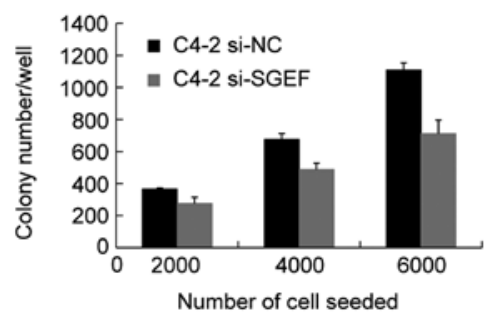

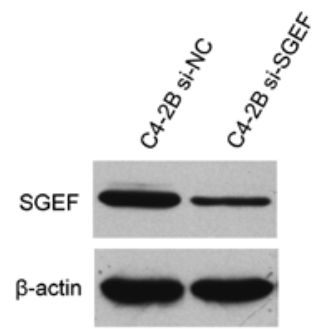
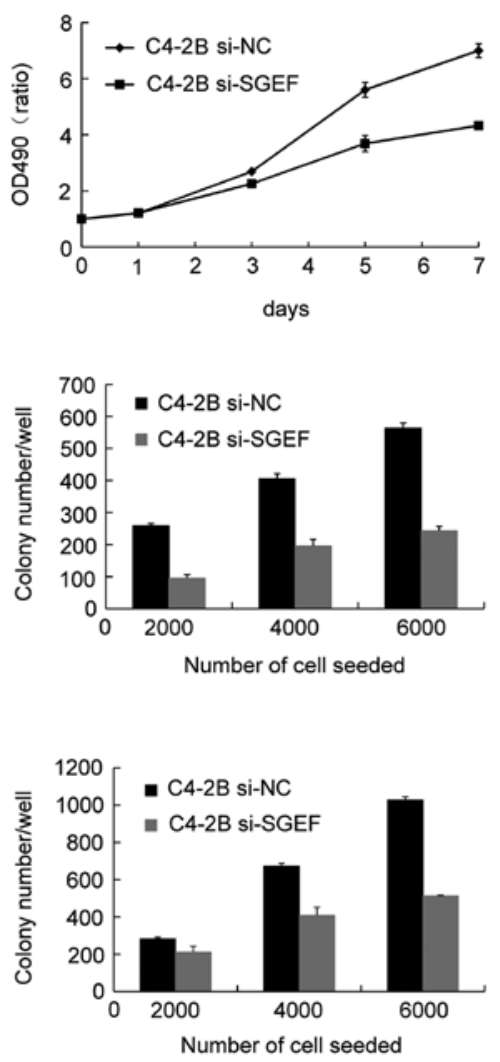

Figure 3. Effects of reduced SGEF expression on prostate cancer cell growth. (A) C4-2 or C4-2B cells were infected with lentivirus containing control or SGEFspecific shRNA for $12 \mathrm{~h}$. At 3 days after infection, proteins were extracted and analyzed using western blotting. (B) Cell growth curve assays. C4-2 or C4-2B cells infected with control or SGEF-specific shRNA were seeded in 96-well plates. At different time-points, the cell number was evaluated using MTT assay. (C) Colony formation assay. 2,000-6,000 C4-2 or C4-2B cells infected with control or SGEF-specific shRNA were seeded in 6-well plates. After 12 days, the colonies containing >50 cells were counted. (D) Soft agar assay. 2,000-6,000 C4-2 or C4-2B cells infected with control or SGEF-specific shRNA were seeded into soft agar medium containing RPMI-1640 medium with $10 \%$ FBS. After 3 weeks, colonies with at least 15 cells were counted. Data are shown as mean \pm SD and are representative of 3 independent experiments.

example, compared with control cells, the colony-formation rate of SGEF-knockdown C4-2 or C4-2B cells was decreased by 40 or $42 \%$, but in the conditions of bicalutamide, the inhibition rate reached up to 81 or $80 \%$, respectively (Fig. 5A and B). Similarly, the soft-agar colony formation rate of $\mathrm{C} 4-2$ or $\mathrm{C} 4-2 \mathrm{~B}$ cells was decreased by 49 or $39 \%$ as a result of decreasing the SGEF expression, whereas the rate was up to 82 or $88 \%$ when treated with bicalutamide (Fig. 5C and D). These results suggested that bicalutamide facilitated the inhibitory effect of reduced SGEF on the growth of C4-2 and C4-2B cells.

Silencing the expression of SGEF inhibits Akt signaling pathway. Abnormal activation of Akt/PKB is significantly associated with the development of prostate cancer. To further elucidate the mechanism mediating SGEF function in prostate cancer progression, we examined the effect of SGEF on Akt phosphorylation in C4-2 and C4-2B cells (Fig. 6). As a result, knockdown of SGEF was observed to suppress the Ser473 phosphorylation of Akt in these two prostate cancer cell lines. These results suggest that AKT may be a downstream target of SGEF and SGEF may mediate tumor cell growth through Akt/PKB signaling pathways.

\section{Discussion}

Previous studies focused on the role of SGEF in cytoskeleton organization, and little attention was given to its roles in cancer. This study is the first on the roles of SGEF in cancer. We found that the expression of SGEF was elevated in human prostate cancer cells and in the epithelial cells of tumor tissues. Downregulation of SGEF inhibited both anchorage-dependent and anchorage-independent growth ability of human prostate 


\section{A}

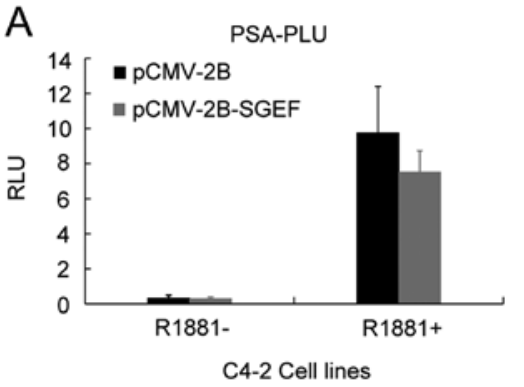

B

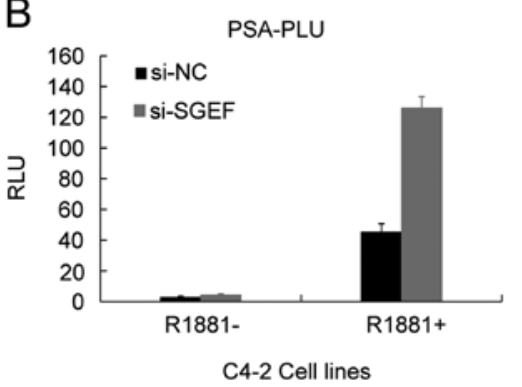

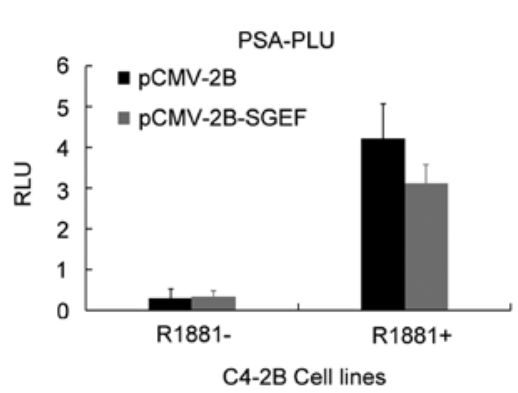

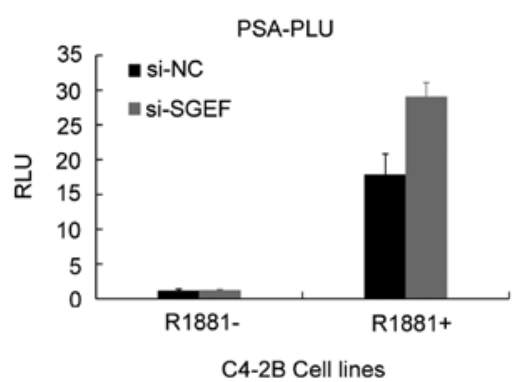

Figure 4. SGEF inhibits AR transcriptional activity. (A) C4-2 or C4-2B cells were co-transfected with 300 ng PSA-luc reporter gene plasmids, $100 \mathrm{ng}$ pCMV-2B or pCMV-2B-SGEF and $30 \mathrm{ng}$ of pCMV- $\beta$-gal plasmids. Cells were treated with or without $0.1 \mathrm{nM} \mathrm{R} 1881$, and analyzed for luciferase activity. Values are mean \pm SD of triplicate measurements. (B) C4-2 or C4-2B cells were co-transfected with $300 \mathrm{ng}$ PSA-luc reporter gene plasmids and $100 \mathrm{ng}$ PGPU6/GFP/Neo-si-NC or PGPU6/GFP/Neo-si-SGEF and $30 \mathrm{ng}$ of pCMV- $\beta$-gal plasmids. Cells were treated and analyzed as in (A).

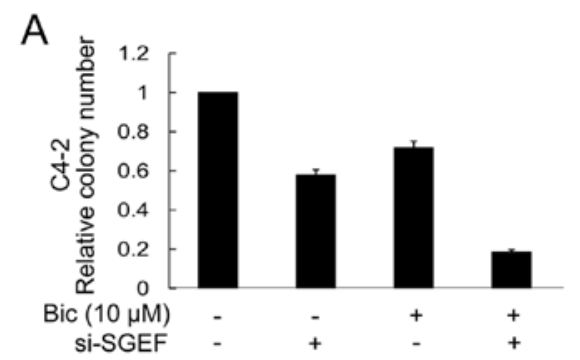

C

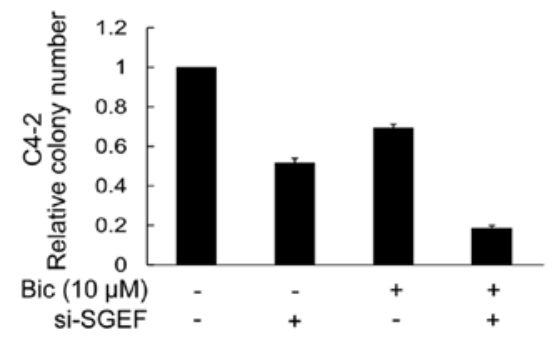

B

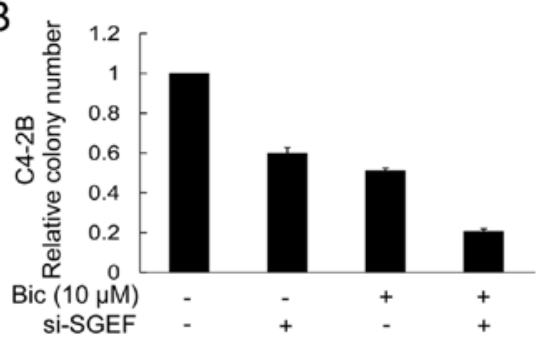

D

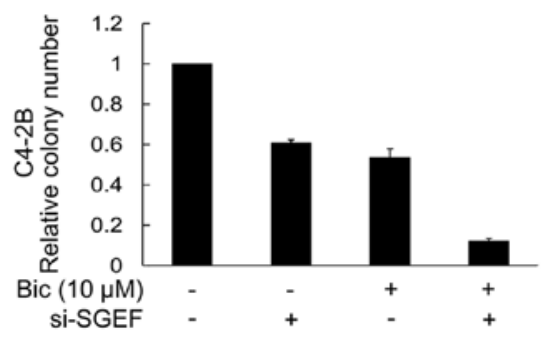

Figure 5. AR antagonist bicalutamide potentiates inhibitory effects of reduced SGEF on the growth of C4-2 and C4-2B cells. (A) Colony formation assay was performed as in Fig. 3C. Cells were treated with or without $10 \mu \mathrm{M}$ bicalutamide. (B) Soft agar assay was performed as in Fig. 3D. Cells were treated with or without $10 \mathrm{nM}$ bicalutamide. Data are shown as mean $\pm \mathrm{SD}$ and are representative of 3 independent experiments.

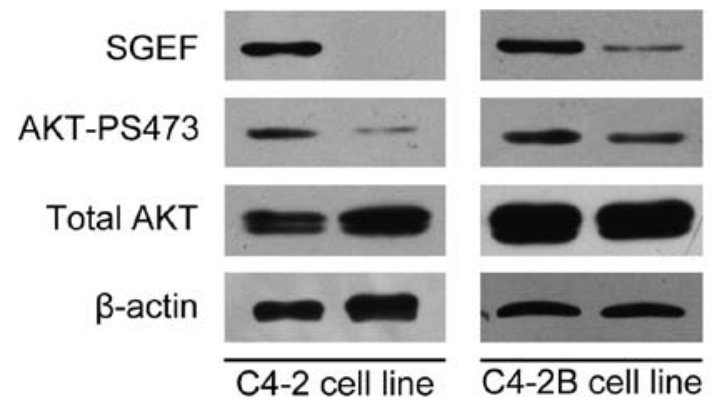

Figure 6. Effects of SGEF on Akt signaling pathway were detected by western blotting. C4-2 or C4-2B cell lines were infected with lentivirus expressing the SGEF si-RNA or NC si-RNA. Whole cell lysate was used for western blot analysis with the indicated antibodies. $\beta$-actin was used as a loading control. 
cancer cells. Moreover, we demonstrated that the inhibitory effect of silenced SGEF on prostate cancer cell growth could be enhanced by the bicalutamide treatment. Taken together, our results indicate that increased expression of SGEF is involved in human prostate cancer progression.

Rho family GTPases are activated in response to a multitude of stimuli and regulate numerous cellular processes including gene expression, cytoskeleton organization, cell proliferation and survival. Because of their central role in regulating diverse signaling pathways and cellular processes, deregulation of the RhoGTPase pathway is assumed to be involved in the development of cancer. In contrast to Ras genes, the typical members of the GTPase super-family, few point mutations were detected in human tumors in RhoGTPases (7). Instead, overexpression and hyperactivity of Rho proteins appear to play a role in human cancer initiation and progression. These facts indicate that regulator proteins of RhoGTPases, especially GEFs, have a crucial role in cancer development. Consistent with this, many GEFs including Dbl, LARG, Vav1 and Ect2 were found as genetic alteration or aberrant expression in several human tumors (8-11). In prostate cancer, several GEFs including Tiam1, PREX1 and Vav3 were reported to be overexpressed and associated with carcinoma progression (12-14). These reports further support our result that SGEF may act as a novel potential oncogene contributing to prostate cancer development.

Enhancement of AR signaling has been thought to be involved in prostate cancer initiation and progression $(15,16)$. Vav3, another GEF similar to SGEF, has been reported to promote prostate cancer cells growth though enhancing AR transactivation (14). To assess whether SGEF functions in the same way, we evaluated the effect of SGEF on the AR transcriptional activity. Unexpectedly, luciferase reporter assays suggested that SGEF repressed AR transactivation. These data indicate that the positive effect of SGEF expression on prostate cancer cell growth is not due to elevated AR activity. Furthermore, these results also provide us an assumption that AR antagonist may further enhance the inhibition of decreased SGEF on the growth of prostate cancer cells though reducing the elevated AR transcriptional activity from silencing SGEF expression. Our subsequent experimental results confirm our hypothesis. In addition, we also found that reducing the expression of SGEF and treatment of the androgen antagonist had a synergistic effect on the suppression of prostate cancer cell growth. These studies provide the possibility that increased SGEF expression is involved in androgen antagonist treatment resistance, which is a critical stage for poor prognosis in prostate cancer.

The PI3K/Akt signaling pathway has a critical role in prostate cancer progression and development. Increased Akt kinase activity correlates with poor prognosis in human prostate cancer and is associated with a hormone therapy-resistant phenotype (17-20). Previous studies have shown that SGEF can activate RhoG, a member of the Rho family of small GTPases (2-4). In addition, RhoG was reported to inhibit anoikis through a phosphatidylinositol 3-kinase-dependent mechanism (21). Overexpression of Vav3, another GEF similar to SGEF, has been reported to elevate the phosphorylation of Akt in prostate cancer cells (14). These studies lead us to examine whether SGEF activate the Akt signaling pathway. Our data show that knockdown of SGEF decreases levels of phosphorylated Akt. Since Akt can regulate a variation of substrates involved in multiple cellular processes, including cell proliferation, cell migration and cell differentiation, we speculate that the activation of Akt signaling pathway may be one of the reason that SGEF contributes to prostate cancer progression. Further studies are necessary to determine how SGEF regulate Akt activity and which substrate of Akt affects cell proliferation as a downstream target of SGEF.

Previous reports have demonstrated that inhibition of PI3K/ Akt signaling pathways enhances AR transcriptional activity (22-25). Additionally, a recent study shows that PI3K-AKTmTOR pathway is dominant over AR signaling in prostate cancer cell growth (26). Data presented here suggest that decreasing the expression of SGEF enhance the AR transactivation, but inhibits the PI3K/Akt signaling. However, in spite of an increase in AR signaling, which is thought to have proliferative effects, the end result of knockdown of SGEF is reduced prostate cancer cell growth which can be further enhanced when AR signaling is blocked. Our results provide a possibility that reduced SGEF increases AR signaling though inhibition of the PI3K/AKT pathway and results in an inhibitory effect on cell growth due to the dominant role of PI3K/AKT pathway in prostate cancer cell growth. Further experiments should be performed to check this hypothesis.

In conclusion, this study reveals that SGEF is overexpressed in human prostate cancer cells and contribute to prostate cancer progression. These data suggest SGEF could be a new potential marker and an efficacious therapeutic target for human prostate cancer.

\section{Acknowledgements}

This study was supported by grants to Jianguang Zhou and Jian Wang from National Natural Science Foundation of China (nos. 30870961 and 81172445).

\section{References}

1. Qi H, Fournier A, Grenier J, Fillion C, Labrie Y and Labrie C: Isolation of the novel human guanine nucleotide exchange factor Src homology 3 domain-containing guanine nucleotide exchange factor and of C-terminal SGEF, an N-terminally truncated form of SGEF, the expression of which is regulated by androgen in prostate cancer cells. Endocrinology 144: 1742-1752, 2003.

2. Ellerbroek SM, Wennerberg K, Arthur WT, et al: SGEF, a RhoG guanine nucleotide exchange factor that stimulates macropinocytosis. Mol Biol Cell 15: 3309-3319, 2004.

3. Patel JC and Galan JE: Differential activation and function of Rho GTPases during Salmonella-host cell interactions. J Cell Biol 175: 453-463, 2006.

4. Van Buul JD, Allingham MJ, Samson T, et al: RhoG regulates endothelial apical cup assembly downstream from ICAM1 engagement and is involved in leukocyte trans-endothelial migration. J Cell Biol 178: 1279-1293, 2007.

5. Jemal A, Siegel R, Ward E, Murray, Xu J and Thun MJ: Cancer statistics. Cancer J Clin 57: 43-66, 2007.

6. Pang B, Zhang H, Wang J, et al: Ubiquitous mitochondrial creatine kinase is overexpressed in the conditioned medium and the extract of LNCaP lineaged androgen independent cell lines and facilitates prostate cancer progression. Prostate 69: 1176-1187, 2009.

7. Schubbert S, Shannon K and Bollag G: Hyperactive Ras in developmental disorders and cancer. Nat Rev Cancer 7: 295-308, 2007.

8. Advani AS and Pendergast AM: Bcr-Abl variants: biological and clinical aspects. Leuk Res 26: 713-720, 2002.

9. Kourlas PJ, Strout MP, Becknell B, et al: Identification of a gene at 11q23 encoding a guanine nucleotide exchange factor: evidence for its fusion with MLL in acute myeloid leukemia. Proc Natl Acad Sci USA 97: 2145-2150, 2002. 
10. Fernandez-Zapico ME, Gonzalez-Paz NC, Weiss E, et al: Ectopic expression of VAV1 reveals an unexpected role in pancreatic cancer tumorigenesis. Cancer Cell 7: 39-49, 2005.

11. Hirata D, Yamabuki T and Miki D: Involvement of epithelial cell transforming sequence-2 oncoantigen in lung and esophageal cancer progression. Clin Cancer Res 15: 256-266, 2009.

12. Engers R, Mueller M, Walter A, Collard JG, Willers R and Gabbert HE: Prognostic relevance of Tiam1 protein expression in prostate carcinomas. Br J Cancer 95: 1081-1086, 2006.

13. Qin J, Xie Y, Wang B, et al: Upregulation of PIP3-dependent Rac exchanger 1 (P-Rex1) promotes prostate cancer metastasis. Oncogene 28: 1853-1863, 2009.

14. Dong ZY, Liu Y, Lu S, et al: Vav3 oncogene is overexpressed and regulates cell growth and androgen receptor activity in human prostate cancer. Mol Endocrinol 20: 2315-2325, 2006.

15. Linja MJ, Savinainen KJ, Saramaki OR, Tammela TL, Vessella RL and Visakorpi T: Amplification and overexpression of androgen receptor gene in hormone-refractory prostate cancer. Cancer Res 61: 3550-3555, 2001.

16. Ruizeveld de Winter JA, Janssen PJ, Sleddens HM, et al: Androgen receptor status in localized and locally progressive hormone refractory human prostate cancer. Am J Pathol 144: 735-746, 1994.

17. Edwards J, Krishna NS, Witton CJ and Bartlett JM: Gene amplifications associated with the development of hormone resistant prostate cancer. Clin Cancer Res 9: 5271-5281, 2003.

18. Zhang H, Wang J, Pang B, et al: PC-1/PrLZ contributes to malignant progression in prostate cancer. Cancer Res 67: 8906-8913, 2007.
19. Nakatani K, Thompson DA, Barthel A, et al: Upregulation of Akt 3 in estrogen receptor deficient breast cancers and androgenindependent prostate cancer lines. J Biol Chem 274: 21528-21532, 1999.

20. Ayala G, Thompson T, Yang G, et al: High levels of phosphorylated form of Akt-1 in prostate cancer and non-neoplastic tissues are strong predictors of biochemical recurrence. Clin Cancer Res 10: 6572-6578, 2004.

21. Yamaki N, Negishi $\mathrm{M}$ and Katoh $\mathrm{H}$ : RhoG regulates anoikis through a phosphatidylinositol 3-kinase-dependent mechanism. Exp Cell Res 313: 2821-2832, 2007.

22. Yang L, Lin HK, Altuwaijri S, Xie S, Wang L and Chang C.: APPL suppresses androgen receptor transactivation via potentiating Akt activity. J Biol Chem 278: 16820-16827, 2003.

23. Lin HK, Yeh S, Kang HY and Chang C: Akt suppresses androgeninduced apoptosis by phosphorylating and inhibiting androgen receptor. Proc Natl Acad Sci USA 98: 7200-7205, 2001.

24. Yang L, Wang L, Lin HK, et al: Interleukin-6 differentially regulates androgen receptor transactivation via PI3K-Akt, STAT3, and MAPK, three distinct signal pathways in prostate cancer cell. Biochem Biophys Res Commun 305: 462-469, 2003.

25. Yang L, Xie S, Jamaluddin MS, et al: Induction of androgen receptor expression by phosphatidylinositol3-kinase/Akt downstream substrate, FOXO3a, and their roles in apoptosis of LNCaP prostate cancer cells. J Biol Chem 280: 33558-33565, 2005.

26. Kaarbø M, Mikkelsen OL, Malerød L, et al: PI3K-AKT-mTOR pathway is dominant over androgen receptor signaling in prostate cancer cells. Cell Oncol 32: 11-27, 2010. 